\title{
Different Ways of Cell-Death: is Death Really Important?
}

\section{Puja Kumari*}

Department of Immunology, UConn Health, School of Medicine, Farmington, USA

*Corresponding Author: Puja Kumari, Department of Immunology, UConn Health, School of Medicine, Farmington, USA.

Received: July 29, 2019; Published: October 01, 2019

DOI: $10.31080 /$ ASMI.2019.02.0389

Cells undergo various ways of death depending on the type, stimulus, and need of the host. By far, various methods of celldeath have been reported such as apoptosis, necrosis, necroptosis, pyroptosis, NETosis, to name a few [1] and among them, necrosis, apoptosis, and pyroptosis have been studied extensively. Interestingly, these cell-deaths differ from each other by the type of deathstimulus, signaling pathways, and its consequences. Necrosis occurs due to injury (mechanical or chemical) to cells, wherein cells get ruptured leading to leakage of cellular contents and eventually a death. This leakage through the necrotic cells stimulates localized inflammation around the affected area. Unlike necrosis, apoptosis is a programmed way of cell-death wherein cells die to turn over the cellular population or remove the infected and altered cells from the body. Apoptosis is a very neat method of cell-death wherein apoptotic cells prevent leakage of the cellular contents and instead package them into small vesicles to release out in the form of apoptotic bodies, which eventually are phagocytosed by nearby phagocytes. Hence, apoptosis does not stimulate inflammation. Coming to the basic question, is cell-death important? One answer is 'No' if the cells are dying due to necrosis. The other answer is 'Yes' if cells are dying due to apoptosis to remove out the infection or toxicity prevailing in them.

Certain intracellular or extracellular agents induce a different type of cell-death than necrosis and apoptosis, wherein small pores are formed in the plasma membrane of the dying cells. These pores allow cytokines and other alarmins to be released out and alarm other cells for certain infection or invasion. These pores grow big and eventually lead to cell-death, followed by systemic inflammation due to exposure of cellular contents to the milieu and cytokine storm. Such type of inflammatory cell-death is known as pyroptosis [2,3]. Conclusively, it indicates that the pyroptosis is an amalgam of necrosis and apoptosis, wherein cells die in a programmed manner and yet release out cellular contents. However, why does a cell undergo pyroptosis when it could safely die with apoptosis? In view of infection, apoptosis clears out intracellular infection without alarming the neighboring cells; however, pyroptosis systemically alarms the cells of an infection. Thus, pyroptosis helps prepare the uninfected cells to fight and clear the infection. At the same time, apoptosis is more global than pyroptosis, which is limited to a few immune cells such as monocytes and macrophages, as well as to endothelial cells [4]. Hence, cell-death is important to maintain a homeostasis in the host.

\section{Bibliography}

1. Galluzzi L., et al. "Molecular mechanisms of cell death: recommendations of the Nomenclature Committee on Cell Death 2018". Cell Death and Differentiation 25 (2018): 486-541.

2. Platnich JM and DA Muruve. "NOD-like receptors and inflammasomes: A review of their canonical and non-canonical signaling pathways". Archives of Biochemistry and Biophysics (2019).

3. Vanaja SK., et al. "Mechanisms of inflammasome activation: recent advances and novel insights". Trends in Cell Biology 25 (2015): 308-315.

4. Cheng KT., et al. "Caspase-11-mediated endothelial pyroptosis underlies endotoxemia-induced lung injury". Journal of Clinical Investigation 127.11 (2017): 4124-4135.

Volume 2 Issue 11 November 2019 (C) All rights are reserved by Puja Kumari. 